\title{
Les prélèvements testiculaires dans les azoospermies sécrétoires : le point de vue du Biologiste de la Reproduction sur la lecture du prélèvement.
}

\author{
J. LORNAGE, S. GISCARD D'ESTAING, H. LEJEUNE, M. BENCHAIB, D. COTTINET, B. SALLE, \\ D. BOULIEU*, V. BIED-DAMON, S. HADJ, J.F. GUERIN
}

\begin{abstract}
Département de Médecine de la Reproduction, * Service de Gynécologie, Hôpital EdouardHerriot, Lyon
\end{abstract}

\section{RESUME}

Le pronostic des infertilités masculines sévères, dont les azoospermies sécrétoires, s'est considérablement amélioré ces dernières années avec la microinjection (ICSI). Le biologiste de la Reproduction participe activement, en collaboration avec le chirurgien à la recherche et l'extraction des spermatozoïdes à partir du tissu testiculaire (TESE).

La présence du biologiste au bloc opératoire est impérative pour guider le chirurgien et éviter des biopsies trop nombreuses. L'extraction des spermatozoïdes au laboratoire se fait le plus souvent par extraction mécanique en dilacérant le tissu en fins lambeaux à l'aide de pinces fines. L'extraction enzymatique à l'aide de collagénase de type IV pourrait être employée dans le cas des azoospermies sécrétoires afin de mieux dissocier les cellules et d'augmenter les chances de récupération de formes matures. La purification des spermatozoïdes sur fraction $50 \%$ de PureSperm permet de limiter la perte en spermatozoïdes sur des prélèvements qui sont souvent moins cellulaires que dans les cas d'azoospermie excrétoire. L'addition de stimulant de la mobilité (telle que la Pentoxifylline) ou la culture in vitro pendant 3 jours dans le cas d'akinésie, permet d'améliorer les résultats en AMP.
La cryopréservation des spermatozoïdes est obligatoire dans la prise en charge de ces cas d'azoospermie, permettant de programmer la TESE de manière asynchrone par rapport à la FIV-ICSI. Les résultats de cryoconservation des spermatozoïdes testiculaires obtenus ces dernières années sont satisfaisants. La congélation de plusieurs fractions spermatiques de petit volume serait à privilégier. Il apparaît important de diminuer les interventions chirurgicales sur le testicule.

Nous présentons brièvement notre expérience sur 36 cas d'azoospermie sécrétoire, dans 13 cas la recherche de spermatozoïdes a été négative soit $36 \%$; c'est le taux qui est retrouvé dans la littérature.

Mots clés : azoospermie sécrétoire - extraction congélation - spermatozoïdes testiculaires

Correspondance : J. Lornage, Département de Médecine de la Reproduction, Hôpital E. Herriot, pavillon L, 5 place d'Arsonval - 69437 LYON Cédex 03 - Tél. 047211 7760 ; Fax. 0472116907.

Communication au XVIlème Congrès de la SALF, 7-9 décembre 2000, Bordeaux. 


\section{INTRODUCTION}

Les azoospermies sécrétoires sont caractérisées par une altération de la production ou de la maturation des spermatozoïdes. Elles peuvent être primaires, les étiologies sont souvent inconnues mais les causes génétiques recherchées en priorité (anomalies chromosomiques, microdélétions du chromosome Y). Elles peuvent être secondaires à certaines pathologies acquises (infections, traumatisme), ou à des traitements (chimio ou radiothérapie).

La classification histologique des azoospermies sécrétoires selon Levin [9], est importante car elle permet de prédire la présence ou non de spermatozoïdes : arrêts de maturation plus ou moins précoces, scléroses tubaires, syndrome des cellules de Sertoli seules, hypoplasie des cellules de la lignée germinale (hypospermatogénèse). Cependant il peut demeurer des foyers isolés de spermatogenèse qui ne sont pas retrouvés à l'examen histologique qui se fait le plus souvent sur une seule biopsie.

Dès 1995, Devroey et al. [5] obtenaient les premières naissances après microinjection de spermatozoïdes obtenus à partir de biopsies testiculaires dans les cas d'azoospermie sécrétoire. La technique d'extraction des spermatozoïdes à partir de biopsie testiculaire (TESE) s'est alors développée et a révolutionné le traitement de l'azoospermie sécrétoire. L'objectif du biologiste de la Reproduction est de retrouver des formes matures en quantité suffisante pour réaliser une fécondation in vitro (FIV) avec microinjection intra cytoplasmique (ICSI). Ce geste est à la fois diagnostique et thérapeutique.

Nous présentons dans une première partie les différentes techniques utilisées pour l'extraction des spermatozoïdes testiculaires dans les cas plus particuliers d'azoospermies sécrétoires puis nous donnons des résultats préliminaires sur notre expérience dans notre équipe. Nous abordons aussi l'intérêt de la congélation des spermatozoïdes testiculaires dans ces cas d'azoospermie sécrétoire.

\section{METHODES DE RECUEIL ET D'EXTRACTION DES SPERMATOZOIDES TESTICULAIRES}

\section{Le temps opératoire}

Les prélèvements testiculaires sont réalisés au bloc opératoire soit de manière asynchrone, soit de manière synchrone couplée à une Assistance Médicale à la Procréation (AMP). Deux grandes techniques chirurgicales sont employées actuellement : biopsies testiculaires multiples à ciel ouvert et les ponctions testiculaires multiples per-cutanées à l'aiguille. Cependant, les techniques de biopsie avec microdissection des tubes séminiferes les plus larges et les plus opaques semblent être de plus en plus privilégiées dans les cas d'azoospermie non-obstructive [14].

La présence du biologiste au bloc opératoire est essentielle, il guide le chirurgien en examinant de manière extemporanée, les prélèvements testiculaires : quelques tubules séminiferres sont prélevés et dilacérés puis examinés sous le microscope. Une biopsie plus large est faite dans le territoire où les spermatozoïdes ont été mis en évidence [13]. De cette manière, 1 à 4 biopsies peuvent être réalisées sur chaque testicule : 2 biopsies au pôle supérieur, 2 biopsies au pôle inférieur (soit une biopsie sur chaque cadran du testicule). Les biopsies sont rincées dans le sérum physiologique afin d'éviter d'avoir trop d'hématies dans nos préparations de spermatozoïdes, puis elles sont placées dans de petits tubes en polypropylène dans un milieu de survie des spermatozoïdes à température d'environ $25^{\circ} \mathrm{C}$. Au bloc opératoire, les biopsies sont dilacérées à l'aide de ciseaux fins : ceci va permettre la sortie des spermatozoïdes pendant le transport vers le laboratoire.

\section{Extraction des spermatozoïdes au laboratoire}

Deux techniques principales d'extraction des spermatozoïdes à partir des biopsies sont utilisées : l'extraction mécanique et l'extraction enzymatique. 
- La technique la plus courante est l'extraction mécanique en dilacérant les tubes séminiferes. Pour augmenter le nombre total de spermatozoïdes qui peut être extrait d'une biopsie testiculaire, 4 méthodes mécaniques de préparation ont été comparées [14] :

1. les lambeaux grossiers de biopsies sont écrasés entre deux lames en verre (technique contrôle),

2. les lambeaux sont plus finement dilacérés à l'aide de deux pinces fines,

3. les lambeaux sont agités à l'aide d'un vortex,

4. les lambeaux sont écrasés à l'aide d'un potter électrique.

De plus, Verheyen et al. [18] utilisaient un tampon de lyse des érythrocytes après dilacération des tubes séminifères, ceci permettant d'améliorer la lecture du prélèvement en diminuant les érythrocytes. Après extraction mécanique, peu de différences ont été mises en évidence entre les 4 techniques : les nombres totaux de spermatozoïdes extraits étaient comparables, il y avait cependant plus de spermatozoïdes vivants après la technique contrôle et la technique de dilacération en fins lambeaux.

- L'extraction enzymatique à l'aide de la collagénase de type IV est employée dans les cas d'hypospermatogénèse sévère lorsqu'aucun ou peu de spermatozoïdes sont retrouvés après l'extraction mécanique [4]. Cette technique enzymatique permet un meilleur rendement en cellules intactes avec des altérations réduites de la membrane cellulaire en comparaison avec les techniques mécaniques. Dans cette étude, les auteurs ont comparé les collagénases type IA et type IV, ils ont obtenu de meilleurs résultats avec la collagénase type IV qui permet une digestion du tissu testiculaire avec un taux de viabilité des cellules germinales plus élevé. L'addition d'élastase à la collagénase type IV n'augmente pas la digestion du tissu testiculaire et donc le taux de récupération de spermatozoïdes à partir des biopsies. De plus, il est préférable de ne pas utiliser une seconde protéase afin de réduire les altérations membranaires des cellules.

\section{Purification des spermatozoïdes à l'aide d'un gradient de discontinu de densité}

Cette étape permet d'isoler les spermatozoïdes de morphologie normale et d'initier leur mobilité.

Dans les cas trop limites en nombre de spermatozoïdes, une technique de lavage seule est réalisée avant l'injection du spermatozoïde dans le cytoplasme de l'ovocyte.

Lorsque le prélèvement est très riche en cellules, il était nécessaire de réaliser un gradient discontinu (95\% - 47,5\%) sur deux couches de Percoll, Cependant une perte importante en spermatozoïdes d'environ $90 \%$, était observé par les auteurs [18]. La méthode mécanique de dilacération en fins lambeaux associée à un gradient de Percoll a donné le meilleur rendement en nombre total de spermatozoïdes mobiles et en pourcentage de formes normales. Ce procédé permet d'isoler une fraction très enrichie en spermatozoïdes. Cependant dans le cas d'azoospermie sécrétoire, les suspensions recueillies sont beaucoup moins riches en cellules et nous ne pouvons pas nous permettre d'avoir une perte en spermatozoïdes aussi importante. Classiquement, dans notre équipe, nous utilisons une couche de PureSperm (JC Diffusion, Scandivian IVF Science, Lyon, France) $50 \%$ qui permet d'isoler les cellules les plus petites et d'enrichir la fraction en spermatozoïdes. De cette manière, la perte en spermatozoïdes est limitée.

L'initiation de la mobilité est réalisée en laissant incuber dans l'étuve à $37^{\circ} \mathrm{C}$ sous $5 \%$ de $\mathrm{CO}_{2}$ pendant 1 à 2 heures. Linjection de spermatozoïdes immobiles entraîne une chute dramatique du taux de fécondation [3]. Dans les cas d'akinésie totale des spermatozoïdes, soit un test hypoosmotique, soit l'addition de stimulant tel que la pentoxifylline, soit la culture in vitro peuvent être proposés.

Le test hypoosmotique peut être employé dans les cas de spermes testiculaires [10]. Un test hypoosmotique simplifié peut être réalisé en diluant de moitié le milieu de culture avec de l'eau désionisée stérile [15]. Aucune mobilité n'était observée après préparation dans 12 cas de spermes testiculaire. Ce test a permis d'ob- 
tenir un taux de fécondation à $30 \%$ et 2 grossesses. Ce test est d'utilisation facile et permet l'obtention de grossesses.

L'utilisation de Pentoxifylline permet d'augmenter rapidement la mobilité des spermatozoïdes testiculaires et d'obtenir des grossesses lorsqu'aucune mobilité n'était obtenue après préparation [8, 11]. Sur 10 échantillons testés, incubés pendant 10 minutes avec $3,6 \mathrm{M}$ de Pentoxifylline, la mobilité des spermatozoïdes était toujours initiée avec une mobilité moyenne de $13,6 \%$ pour les échantillons de spermatozoïdes testiculaires "frais " ou après congélation [17]. Les auteurs n'ont pas montré de différence sur l'évolutivité des embryons et l'obtention de grossesses entre le groupe de spermatozoïdes épididymaires ou testiculaires immobiles, avec une mobilité initiée par la Pentoxifylline et le groupe témoin qui montre une mobilité spontanée après préparation. Cependant, afin d'éviter l'addition de stimulant dans les préparations de spermatozoïdes testiculaires en vue d'ICSI, d'autres équipes préconisent la culture in vitro à $37^{\circ} \mathrm{C}$ pendant 48 à 96 heures pour initier la mobilité de manière comparable à l'action de la Pentoxifylline pendant 30 minutes [2]. Cette technique pourrait être envisagée lorsque les ovocytes ne sont pas immédiatement prêts pour la fécondation.

De plus, dans les cas d'azoospermie obstructive, la culture in vitro sur 3 jours apparaît donnée des résultats satisfaisants en terme de qualité de l'ADN avec une augmentation des spermatozoïdes montrant un ADN double brin de $22 \%$ à $50 \%$ en utilisant une coloration à l'Acridine Orange. Cependant dans le cas d'azoospermie sécrétoire, il n'y aurait pas d'amélioration [6].

Dans un programme d'Assistance Médicale à la Procréation, l'addition de stimulant apparaît être la solution la plus rapide pour obtenir des spermatozoïdes mobiles. Dans le cas d'azoospermie sécrétoire, la culture in vitro n'a pas prouvé son efficacité.

\section{METHODES DE CRYOCONSERVA- TION DES SPERMATOZOIDES TESTICULAIRES}

Le but de la congélation des spermatozoïdes est d'éviter des ponctions testiculaires itératives dans le cadre d'une Assistance Médicale à la Procréation. La fraction excédentaire de spermatozoïdes isolés lorsqu'une FIV a été programmée en synchrone ou la fraction totale de spermatozoïdes dans les cas asynchrones sont congelés selon le protocole standard en utilisant le glycérol comme cryoprotecteur. Plusieurs équipes ont montré des résultats tout à fait comparables en taux de fécondation, de segmentation et en obtention de grossesses en utilisant des spermes testiculaires soit " frais " soit congelés dans le cas d'azoospermie non obstructive $[12,3,7]$. Afin de réduire la quantité de spermatozoïdes congelés dans une même unité, il a été proposé de congeler les spermatozoïdes dans des zones pellucides d'ovocytes vides [19]. Deux naissances de jumeaux et une grossesse unique ont été obtenues dans cette étude.

\section{RESULTATS}

Trente six biopsies testiculaires, dans le cas d'azoospermie sécrétoire, ont été programmées dans notre centre : 26 cas purement sécrétoires et 10 cas d'origine mixte, à la fois excrétoires et sécrétoires.

Dans 19 cas (53\%), la recherche de spermatozoïdes a été positive et une FIV avec microinjection, a pu être réalisée soit de manière asynchrone soit de manière synchrone.

Dans 13 cas (36\%), la recherche de spermatozoïdes a été négative ; une seule a été réalisée en synchrone. Dans ce dernier cas, une biopsie avec étude anatomopathologique avait été faite les années précédentes et montrait la présence de rares spermatozoïdes dans quelques tubes séminifères.

Dans 3 cas, alors que l'indication de TESEICSI avait été initialement retenue, les couples 
ont renoncé à l'exploration testiculaire après avoir vu l'ensemble de l'équipe y compris le biologiste. D'autres cas d'abandons, et orientation vers l'adoption ou le don de sperme ont lieu à la suite de l'investigation andrologique initiale.

Dans un dernier cas, l'exploration n'a pas encore été réalisée.

Nous avons obtenu 3 grossesses dont 2 grossesses gémellaires sur 20 cycles réalisés. La dernière grossesse a été obtenue à partir de spermatozoïdes testiculaires congelés.

Dans les 13 cas d'absence de spermatozoïdes au cours de la TESE, nous avions 6 cas d'involution sertolienne totale, un syndrome de cellules de Sertoli seules, 6 blocages au stade spermatocytaire. Dans 3 cas, il existait une microdélétion dans une des régions $\mathrm{AZF}$ et dans un cas, une anomalie chromosomique : translocation $9 ; 19$.

\section{CONCLUSION}

Il n'existe pas de critères prédictifs de la présence de spermatozoïdes dans les tubes séminifères qui permettraient de trancher entre les prélèvements synchrones d'une AMP ou asynchrones. La biopsie testiculaire, avec extraction des spermatozoïdes reste la technique la plus fiable pour décider d'une ICSI. Dans les cas d'azoospermie sécrétoire (FSH élevée, volume testiculaire réduit), la réalisation de la ponction testiculaire avec une étape de cryoconservation des spermatozoïdes est actuellement privilégiée dans notre équipe.

Cette technique exige une étroite collaboration entre le chirurgien et le biologiste ainsi que l'équipe gynécologique lorsqu'une AMP est programmée en synchrone. Il est très important d'éviter de répéter ces biopsies testiculaires. Tash et Schlegel [16] ont montré que la TESE entraîne une diminution du volume des tubes séminifêres autour du site de la biopsie. Un intervalle minimum de 6 mois [1] est préconisé entre deux biopsies testiculaires pour une bonne cicatrisation. De plus, au cours de la chirurgie pour l'extraction testiculaire des spermatozoïdes (TESE), il faut réduire au maximum la perte de tissu.

\section{REFERENCES}

1- AMER M., HAGGAR S.E., MOUSTAFA T., et al. : Testicular sperm extraction : impact of testicular histology on outcome, number of biopsies to be performed and optimal time for repetition. Hum. Reprod., 1999, $14: 3030-3034$.

2- ANGELOPOULOS T., ADLER A., KREY L., et al. : Enhancement or initiation of testicular sperm motility by in vitro culture of testicular tissue. Fertil. Steril., 1999, $71: 240-243$.

3- BEN-YOSEF D., YOGEV L., HAUSER R., YAVETZ $H$., et al. : Testicular sperm retrieval and cryopreservation prior to initiating ovarian stimulation as the first line approach in patients with non-obstructive azoospermia. Hum. Reprod, 1999, 14 : 1794-1801.

4- CRABBE E., VERHEYEN G., SILBER S., et al. : Enzymatic digestion of testicular tissue may rescue the intracytoplasmic sperm injection cycle in some patients with non-obstructive azoospermia. Hum. Reprod., 1998, 10 : 2791-2796.

5- DEVROEY P., LIU J., NAGY P., et al. : Pregnancies after testicular sperm extraction and intracytoplasmic sperm injection in non obstructive azoospermia. Hum. Reprod., 1995, $10: 1457-1460$.

6- EMILIANI S., VAN DEN BERGH M., VANNIN A.S., et al. : Evidence of reduced single-stranded testicular sperm DNA from obstructive azoospermic men after 3 days of in-vitro culture. Hum. Reprod., 2001, 16 : 1200-1203.

7- HUANG F.J., CHANG S.Y., TSAI M.Y., et al. : Clinical implications of intracytoplasmic sperm injection using cryopreserved testicular spermatozoa from men with azoospermia. J. Reprod. Med., 2000, 45: 310-316.

8- KOHN F.M., SCHROEDER-PRINTZEN I., EL MULLA K.F., et al. : Andrological work-up of patients undergoing microsurgical epididymal sperm aspiration or testicular sperm extraction. Andrologia, 1996, 28, Suppl 1 : 77-81.

9- LEVIN H.S. : Testicular biopsy in the study of male infertility. Its current usefulness, histologic techniques, and prospects for the future. Hum. Pathol., $1979,10: 569-584$.

10- LIN M.H., MORSHEDI M., SRISOMBUT C., et al. : Plasma membrane integrity of cryopreserved human sperm : an investigation of the results of the hypoosmotic swelling test, the water test, and eosin-Y staining. Fertil. Steril., 1998, $70: 1148-1155$.

11- NODAR F., DE VINCENTIIS S., OLMEDO S.B., et al. : Birth of twin males with normal karyotype after intracytoplasmic sperm injection with use of testicular spermatozoa from a nonmosaic patient with Klinefelter's syndrome. Fertil. Steril., 1999 Jun ; 71 : 1149-1152. 
12- OATES R.D., MULHALL J., BURGESS C., et al. : Fertilization and pregnancy using intentionally cryopreserved testicular tissue as the sperm source for intracytoplasmic sperm injection in 10 men with nonobstructive azoospermia. Hum. Reprod., 1997, 12 : 734-739.

13- OSTAD M., LIOTTA D., YE Z., SCHLEGEL N.P. : Testicular sperm extraction for non-obstructive azoospermia : result of a multibiopsy approch with optimized tissu dispersion. Urology, 1998, $52: 4: 692-$ 696.

14- SCHLEGEL N.P. : Testicular sperm extraction : micro-dissection improves sperm yield with minimal tissue excision. Hum. Reprod., 1999, 14 : 131-135.

15- SALLAM H., FARRAG A., AGAMEYA., et al. : The use of a modified hypo-osmotic swelling test for the selection of viable ejaculated and testicular immotile spermatozoa in ICSI. Hum. Reprod., 2001, 16 : 272276.

16- TASH J.A., SCHLEGEL P.N. : Histologic effects of testicular sperm extraction on the testicle in men with nonobstructive azoospermia. Urology, 2001, 57 : 334-337.

17- TERRIOU P., HANS E., GIORGETTI C., et al. : Pentoxifylline initiates motility in spontaneously immotile epididymal and testicular spermatozoa and allows normal fertilization, pregnancy, and birth after intracytoplasmic sperm injection. J. Assist. Reprod. Genet., 2000, 17 : 194-199.

18- VERHEYEN G., DE CROO I., TOURNAYE H., et al. : Comparison of four mechanical methods to retrieve spermatozoa from testicular tissue. Hum. Reprod., 1995, $10: 2956-2956$.

19- WALMSLEY. R., COHEN J., FERRARA-CONGEDO T., et al. : The first births and ongoing pregnancies associated with sperm cryopreservation within evacuated egg zonae. Hum. Reprod., 1998, 13, Suppl 4 : 61-70.

\section{ABSTRACT}

The testicular sperm extraction in men with nonobstructive azoospermia: the reproduction biologist's point of view on interpretation of biopsies.

LORNAGE J., GISCARD D'ESTAING S., LEJEUNE H., BENCHAIB M., COTTINET D., SALLE B., BOULIEU D., HADJ S., GUERIN J.F.

The prognosis of severe male sterility (nonobstructive azoospermia) has considerably improved over recent years with the intro- duction of ICSI. The human reproduction biologist actively collaborates with the surgeon in the search for and extraction of sperm from testicular tissue (TESE).

The presence of the biologist during surgery is mandatory to guide exploration and to avoid an excessive number of biopsies. Sperm extraction is performed in the laboratory by mechanical extraction with fine forceps. Enzymatic extraction with collagenase IV can be used in cases of nonobstructive azoospermia to optimize tissue dispersion and to improve retrieval of mature cells. The use of only one fraction (50\%) of Pure Sperm (JC Diffusion, Lyon France) limits the loss of spermatozoa. To optimize the results in cases of akinesia, it may be useful to induce sperm motility by pentoxifylline or perform in vitro culture for three days.

Sperm cryopreservation is compulsory in the case of nonobstructive azoospermia. This allows programming of TESE for a different time from that of ICSI. All results obtained with cryopreserved testicular sperm, in recent years, are encouraging. It is also recommended to freeze several small fractions of sperm in order to limit the number of surgical procedures on the testicle. This article presents the results of our experience in 36 cases of nonobstructive azoospermia. Extraction was negative in 13 cases $(36 \%)$, similar to the rate reported in the literature.

Key-Words: Nonobstructive azoospermia - extraction - cryopreservation - testicular sperm 